Pacific Journal of Mathematics

CHARACTERISTIC DIRECTION FOR EQUATIONS OF 


\title{
CHARACTERISTIC DIRECTION FOR EQUATIONS OF MOTION OF NON-NEWTONIAN FLUIDS
}

\author{
J. L. ERICKSEN
}

1. Introduction. According to the Reiner-Rivlin theory of nonNewtonian fluids, ${ }^{1}$ the stress tensor $t_{j}^{i}$ is given in terms of the rate of strain tensor $d_{j}^{i}$ by relations of the form

$$
t_{j}^{i}=-p \delta_{j}^{i}+\mathscr{F}_{1} d_{j}^{i}+\mathscr{F}_{2} d_{k}^{i} d_{j}^{k},
$$

where $p$ is an arbitrary hydrostatic pressure, the $\mathscr{F}$ 's are essentially arbitrary differentiable functions of

$$
\mathrm{II}=-\frac{1}{2} d_{j}^{i} d_{i}^{j}, \quad \mathrm{III}=\operatorname{det} d_{j}^{i},
$$

and $d_{j}^{i}$ satisfies the incompressibility condition

$$
d_{i}^{i}=0 \text {. }
$$

The tensors $d_{j}^{i}$ and $t_{j}^{i}$ are both symmetric.

It is known [2] that the characteristic directions of the corresponding equations of motion are the unit vectors $\nu_{i}$ satisfying

$$
F\left(\nu_{i}\right) \equiv 2 U^{2}+2 U U_{i}^{i}+\left(U_{i}^{i}\right)^{2}-U_{j}^{i} U_{i}^{j}=0,
$$

where

$$
\begin{aligned}
U= & \mathscr{F}_{1}+\mathscr{F}_{2} \mu^{i} \nu_{i}, \\
U_{j}^{i}= & \mathscr{F}_{2}\left(d_{j}^{i}-\nu^{i} \mu_{j}\right)+2\left(\mu^{i}-\nu^{i} \mu_{k} \nu^{k}\right)\left(\mu^{m} d_{m j} \frac{\partial \mathscr{F}_{1}}{\partial \mathrm{III}}-\mu_{j} \frac{\partial \mathscr{F}_{1}}{\partial \mathrm{II}}\right) \\
& +2\left(d_{m}^{i} \mu^{m}-\nu^{i} \mu_{m} \mu^{m}\right)\left(\mu^{n} d_{n j} \frac{\partial \mathscr{F}_{2}}{\partial \mathrm{III}}-\mu_{j} \frac{\partial \mathscr{F}_{2}}{\partial \mathrm{II}}\right), \\
\mu_{i}= & d_{i j} \nu^{j} .
\end{aligned}
$$

Since $F\left(\nu_{i}\right)$ is a continuous function of $\nu_{i}$ on the compact set $\nu_{i} \nu^{i}=1$, a necessary and sufficient condition that no real characteristic directions exist is that $F\left(\nu_{i}\right)$ be of one sign for all unit vectors. Using this fact, we obtain simpler necessary conditions which are shown to be sufficient when $\mathscr{F}_{2} \equiv 0$.

2. Necessary conditions. Let $d_{1}, d_{2}$ and $d_{3}$ denote the eigenvalues of $d_{j}^{i}$. From (3),

Received April 12, 1957.

1 This theory was proposed independently by Reiner [4] for compressible fluids, by Rivlin [5] for incompressible materials. We treat the latter case. 


$$
d_{1}+d_{2}+d_{3}=0 .
$$

We restrict our attention to unit vectors $\nu_{i}$ which are perpendicular to an eigenvector of $d_{j}^{i}$ and note that $F\left(\nu_{i}\right)$, being a continuous function of $\nu_{i}$, must be of one sign for all unit vectors in order that no real characteristic directions exist. Given any unit vector $\nu_{i}$ perpendicular to an eigenvector $e_{i}$ corresponding to $d_{3}$, we may introduce a rectangular Cartesian coordinate system such that, at a point, $\nu_{i}$ is parallel to the positive $x^{1}$-axis and $e_{i}$ is parallel to the $x^{3}$-axis. Then

$$
\begin{gathered}
\nu_{i}=\delta_{i 1}, d_{13}=d_{23}=d_{1 i} d_{3}^{i}=d_{21} d_{3}^{i}=0, \\
2 d_{12}=\left(d_{1}-d_{2}\right) \sin 2 \phi, d_{33}=d_{3},
\end{gathered}
$$

where $\phi$ is the angle between $\nu_{i}$ and an eigenvector corresponding to $d_{1}$. Making these substitutions in $F\left(\nu_{i}\right)$, given by (4), we obtain, by a routine calculation,

$$
\begin{gathered}
F\left(\nu_{i}\right)=2\left[\mathscr{F}_{1}-\mathscr{F}_{2} d_{2}\right]\left\{\mathscr{F}_{1}-\mathscr{F}_{2} d_{3}-\frac{1}{2}\left(d_{1}-d_{2}\right)^{2} \sin ^{2} 2 \phi\left[\frac{\partial \mathscr{F}_{1}}{\partial \mathrm{II}}\right.\right. \\
\left.\left.-d_{3} \frac{\partial \mathscr{F}_{2}}{\partial \mathrm{II}}+d_{3} \frac{\partial \mathscr{F}_{1}}{\partial \mathrm{III}}-d_{3}^{2} \frac{\partial \mathscr{F}_{2}}{\partial \mathrm{III}}\right]\right\},
\end{gathered}
$$

which must be of one sign for all real angles $\phi$. This is clearly true if and only if it is of the same sign for $\phi=0$ and $\phi=\pi / 4$. That is, either

$$
\left[\mathscr{F}_{1}-\mathscr{F}_{2} d_{2}\right]\left[\mathscr{F}_{1}-\mathscr{F}_{2} d_{3}\right]>0
$$

and

$$
\begin{aligned}
{\left[\mathscr{F}_{1}-\mathscr{F}_{2} d_{2}\right]\left\{\mathscr{F}_{1}\right.} & -\mathscr{F}_{2} d_{3}-\frac{1}{2}\left(d_{1}-d_{2}\right)^{2}\left[\frac{\partial \mathscr{F}_{1}}{\partial \mathrm{II}}\right. \\
& \left.\left.-d_{3} \frac{\partial \mathscr{F}_{2}}{\partial \mathrm{II}}+d_{3} \frac{\partial \mathscr{F}_{1}}{\partial \mathrm{II}}-d_{3}^{2} \frac{\partial \mathscr{F}_{2}}{\partial \mathrm{II}}\right]\right\}>0,
\end{aligned}
$$

or (7) and (8) hold simultaneously with the inequalities reversed. By similarly analyzing the cases where $\nu_{i}$ is perpendicular to eigenvectors of $d_{j}^{i}$ corresponding to $d_{1}$ and $d_{2}$, we conclude that either

$$
\left[\mathscr{F}_{1}-\mathscr{F}_{2} d_{i}\right]\left[\mathscr{F}_{1}-\mathscr{F}_{2} d_{j}\right]>0
$$

and

$$
\begin{aligned}
{\left[\mathscr{F}_{1}-\mathscr{F}_{2} d_{j}\right]\left\{\mathscr{F}_{1}\right.} & -\mathscr{F}_{2} d_{k}-\frac{1}{2}\left(d_{i}-d_{j}\right)^{2}\left[\frac{\partial \mathscr{F}_{1}}{\partial \mathrm{II}}\right. \\
& \left.\left.-d_{k} \frac{\partial \mathscr{F}_{2}}{\partial \mathrm{II}}+d_{k} \frac{\partial \mathscr{F}_{1}}{\partial \mathrm{III}}-d_{k}^{2} \frac{\partial \mathscr{F}_{2}}{\partial \mathrm{III}}\right]\right\}>0 \quad(i, j, k \neq),
\end{aligned}
$$


or

$$
\left[\mathscr{F}_{1}-\mathscr{F}_{2} d_{i}\right]\left[\mathscr{F}_{1}-\mathscr{F}_{2} d_{j}\right]<0
$$

and (10) holds with the inequality reversed. Now (11) cannot hold for all $i$ and $j$, so this possibility is ruled out. We thus have

THEOREM 1. A necessary and sufficient condition that no real characteristic directions exist is that $F\left(\nu_{i}\right)>0$; in order that there exist no real characteristic directions perpendicular to an eigenvector of $d_{j}^{i}$, it is necessary and sufficient that the inequalities (9) and (10) hold.

For (9) and (10) to hold, it is necessary and sufficient that either

$$
\mathscr{F}_{1}-\mathscr{F}_{2} d_{i}>0
$$

and

$$
\mathscr{F}_{1}-\mathscr{F}_{2} d_{k}-\frac{1}{2}\left(d_{i}-d_{j}\right)^{2}\left[\frac{\partial \mathscr{F}_{1}}{\partial \mathrm{II}}-d_{k} \frac{\partial \mathscr{F}_{2}}{\partial \mathrm{II}}+d_{k} \frac{\partial \mathscr{F}_{1}}{\partial \mathrm{II}}-d_{k}^{2} \frac{\partial \mathscr{F}_{2}}{\partial \mathrm{III}}\right]>0
$$

or

$$
\mathscr{F}_{1}-\mathscr{F}_{2} d_{i}<0
$$

and

$$
\mathscr{F}_{1}-\mathscr{F}_{2} d_{k}-\frac{1}{2}\left(d_{i}-d_{j}\right)^{2}\left[\frac{\partial \mathscr{F}_{1}}{\partial \mathrm{II}}-d_{k} \frac{\partial \mathscr{F}_{2}}{\partial \mathrm{II}}+d_{k} \frac{\partial \mathscr{F}_{1}}{\partial \mathrm{III}}-d_{k}^{2} \frac{\partial \mathscr{F}_{2}}{\partial \mathrm{III}}\right]<0
$$

3. Equivalent conditions. Let $t_{i}$ denote the eigenvalues of the stress tensor corresponding to the eigenvalue $d_{i}$ of $d_{m n}$ so that from (1),

$$
t_{i}=-p+\mathscr{F}_{1} d_{i}+\mathscr{F}_{2} d_{i}^{2} \text {. }
$$

Using (5),

$$
\begin{aligned}
t_{i}-t_{j} & =\left[\mathscr{F}_{1}+\mathscr{F}_{2}\left(d_{i}+d_{j}\right)\right]\left(d_{i}-d_{j}\right) \\
& =\left[\mathscr{F}_{1}-\mathscr{F}_{2} d_{k}\right]\left(d_{i}-d_{j}\right)
\end{aligned}
$$

From (2) and (5),

$$
\begin{aligned}
& \mathrm{II}=-\frac{1}{2}\left(d_{1}^{2}+d_{2}^{2}+d_{3}^{2}\right)=-\frac{1}{4}\left(d_{i}-d_{j}\right)^{2}-\frac{3}{4} d_{k}^{2}, \\
& \mathrm{III}=d_{1} d_{2} d_{3}=\frac{1}{4} d_{k}\left[d_{k}^{2}-\left(d_{i}-d_{j}\right)^{2}\right]
\end{aligned}
$$

Using (16) and (17) to express $t_{i}-t_{j}$ as a function of $d_{i}-d_{j}$ and $d_{k}(i, j, k \neq)$, we calculate 
(18)

$$
\begin{aligned}
& \left.\frac{\partial\left(t_{i}-t_{j}\right)}{\partial\left(d_{i}-d_{j}\right)}\right|_{a_{k}=\text { const. }} \\
= & \mathscr{F}_{1}-\mathscr{F}_{2} d_{k}-\frac{1}{2}\left(d_{i}-d_{j}\right)^{2}\left[\frac{\partial \mathscr{F}_{1}}{\partial \mathrm{II}}-d_{k} \frac{\partial \mathscr{F}_{2}}{\partial \mathrm{II}}+d_{k} \frac{\partial \mathscr{F}_{1}}{\partial \mathrm{III}}-d_{k}^{2} \frac{\partial \mathscr{F}_{2}}{\partial \mathrm{III}}\right] .
\end{aligned}
$$

From (12), (13), (14), (15), (16), (18) and Theorem 1, we have

THEOREM 2. When the eigenvalues of $d_{j}^{i}$ are all unequal, a necessary and sufficient condition that there exist no real characteristic direction perpendicular to an eigenvector of $d_{j}^{i}$ is that either

$$
\left(t_{i}-t_{j}\right) /\left(d_{i}-d_{j}\right)>0 \text { and } \partial\left(t_{i}-t_{j}\right) /\left.\partial\left(d_{i}-d_{j}\right)\right|_{d_{k}=\text { const. }}>0 \text {, }
$$

or

$$
\left(t_{i}-t_{j}\right) /\left(d_{i}-d_{j}\right)<0 \quad \text { and } \quad \partial\left(t_{i}-t_{j}\right) /\left.\partial\left(d_{i}-d_{j}\right)\right|_{d_{k}=\text { const. }}<0 \quad(i, j, k \neq) .
$$

When (12) holds, the stress power $\Phi$, given by

$$
3 \Phi=3 t_{j}^{i} d_{j}^{i}=\left(t_{1}-t_{2}\right)\left(d_{1}-d_{2}\right)+\left(t_{2}-t_{3}\right)\left(d_{2}-d_{3}\right)+\left(t_{3}-t_{1}\right)\left(d_{3}-d_{1}\right)
$$

is negative, a possibility which many writers exclude on thermodynamic grounds.

4. The case $\mathscr{F}_{2} \equiv 0$. When $\mathscr{F}_{2} \equiv 0, \mathscr{F}_{1} \neq 0$, the characteristic equation (4) has been shown [2] to reduce to

$$
G\left(\nu_{i}\right) \equiv \mathscr{F}_{1}+A^{i} B_{i}=0,
$$

where

$$
\begin{aligned}
& A^{i}=2\left(\mu^{i}-\nu^{i} \mu_{k} \nu^{k}\right), \\
& B_{i}=\mu^{m} d_{m i} \frac{\partial \mathscr{F}_{1}}{\partial \mathrm{III}}-\mu_{i} \frac{\partial \mathscr{F}_{1}}{\partial \mathrm{II}} .
\end{aligned}
$$

In fact, $F\left(\nu_{i}\right)=2 \mathscr{F}_{1} G\left(\nu_{i}\right)$. When $\mathscr{F}_{2}=0, \mathscr{F}_{1}=0$, every direction is characteristic, a case which we exclude. Using the Hamilton-Cayley theorem,

$$
d_{j}^{i} d_{k}^{j} d_{m}^{k}=\mathrm{III} \delta_{m}^{i}-\mathrm{II} d_{m}^{i},
$$

we can reduce (19) to the form

$$
G(\alpha, \beta) \equiv \mathscr{F}_{1}+2(\mathrm{III}-\mathrm{II} \alpha-\beta \alpha) \frac{\partial \mathscr{F}_{1}}{\partial \mathrm{III}}+2\left(\alpha^{2}-\beta\right) \frac{\partial \mathscr{F}_{1}}{\partial \mathrm{II}}=0,
$$

where

$$
\alpha=\mu_{i} \nu^{i}=d_{i \nu^{2} \nu^{i} \nu^{j}}, \quad \beta=\mu^{i} \mu_{i}=d_{k}^{i} d_{i \nu^{2} \nu^{k} \nu^{m}} .
$$


Now (21) is a mapping of the unit sphere $\nu_{i} \nu^{i}=1$ onto a region $R$ in the $\alpha-\beta$ plane. The conditions

$$
\begin{aligned}
& \frac{\partial G}{\partial \alpha}=-2(\mathrm{II}+\beta) \frac{\partial \mathscr{F}_{1}}{\partial \mathrm{III}}+4 \alpha \frac{\partial \mathscr{F}_{1}}{\partial \mathrm{II}}=0, \\
& \frac{\partial G}{\partial \beta}=-2 \alpha \frac{\partial \mathscr{F}_{1}}{\partial \mathrm{III}}-2 \frac{\partial \mathscr{F}_{1}}{\partial \mathrm{II}}=0, \\
& \pm d^{2} G= \pm 4\left[\frac{\partial \mathscr{F}_{1}}{\partial \mathrm{II}} d \alpha^{2}-\frac{\partial \mathscr{F}_{1}}{\partial \mathrm{III}} d \alpha d \beta\right] \geqq 0 \text { for all } d \alpha, d \beta,
\end{aligned}
$$

must be satisfied at any interior point of $R$ at which $G$ is a maximum or minimum. These conditions cannot be satisfied unless $\partial \mathscr{F}_{1} / \partial \mathrm{II}=$ $\partial \mathscr{F}_{1} / \partial \mathrm{III}=0$, in which case $G\left(\nu_{i}\right)$ is independent of $\nu_{i}$, and $\mathscr{F}_{1} \neq 0$ is then necessary and sufficient that there exist no real characteristics. From the implicit function theorem, values of $\nu_{i}$ corresponding to boundary points of $R$ are such that the equations

$$
d \alpha=2 d_{i j} \nu^{i} d \nu^{j}, \quad d \beta=2 d_{k}^{i} d_{i m^{2}}{ }^{k} d \nu^{m}, \quad 0=\nu_{i} d \nu^{i}
$$

do not admit a unique solution for $d_{2}{ }^{i}$ in terms of $d \alpha$ and $d \beta$. We thus have

THEOREM 3. Maximum and minimum values of $G\left(\nu_{i}\right)$, hence of $F\left(\nu_{i}\right)$, hence of $F\left(\nu_{i}\right)$, occur only at values of $\nu_{i}$ such that the vectors $\nu_{i}, d_{i j} \nu^{j}$ and $d_{i}^{k} d_{k m} \nu^{m}$ are linearly dependent or, equivalently, at values such that the determinant $D$ of these three vectors vanishes.

Whatever be the unit vector $\nu_{i}$, we can always choose rectangular Cartesian coordinates such that, at a point, $\nu_{i}=\delta_{i 1}, d_{23}=0$. The condition $D=0$ then reduces to

$$
\begin{aligned}
0 & =\left|\begin{array}{ccc}
1 & 0 & 0 \\
d_{11} & d_{21} & d_{31} \\
d_{11}^{2}+d_{12}^{2}+d_{13}^{2} & d_{21}\left(d_{11}+d_{22}\right) & d_{31}\left(d_{11}+d_{33}\right)
\end{array}\right| \\
& =d_{21} d_{31}\left(d_{33}-d_{22}\right) .
\end{aligned}
$$

If $d_{21}=0\left(d_{31}=0\right), \delta_{i 2}\left(\delta_{i 3}\right)$ is an eigenvector of $d_{i j}$. If $d_{21} d_{31} \neq 0, d_{33}=d_{22}$, the vector with components $\left(0, d_{31},-d_{21}\right)$ is an eigenvector of $d_{i j}$, whence follows

THEOREM 4. The vectors $\nu_{i}, d_{i j^{\nu}} \nu^{j}, d_{i}^{k} d_{k m} \nu^{m}$ can be linearly dependent only when $\nu_{i}$ is perpendicular to an eigenvector of $d_{j}^{i}$.

Theorems 3 and 4 imply that, when $\mathscr{F}_{2} \equiv 0$, we will have $F\left(\nu_{i}\right)>0$ for all unit vectors $\nu_{i}$ if and only if $F\left(\nu_{i}\right)>0$ for each unit vector $\nu_{i}$ which is perpendicular to an eigenvector of $d_{j}^{i}$. From Theorem 1, we then deduce 
THEOREM 5. When $\mathscr{F}_{2} \equiv 0$, a necessary and sufficient condition that there exist no real characteristic directions is that the inequalities (9) and (10) hold.

\section{REFERENCES}

1. M. Baker and J. L. Ericksen, Inequalities restricting the form of the stress-deformation relations for isotropic elastic solids and Reiner-Rivlin fluids, J. Wash. Acad. Sci. 44 (1954), 33-35.

2. J. L. Ericksen, Characteristic surfaces of the equations of motion for non-Newtonian fluids, ZAMP 4 (1953), 260-267.

3. - A consequence of inequalities proposed by Baker and Ericksen, J. Wash. Acad. Sci. 45 (1955), 268.

4. M. Reiner, A mathematical theory of dilatancy, Amer. J. Math. 67 (1945), 350-362.

5. R. S. Rivlin, The hydrodynamics of non-Newtonian fluids I., Proc. Roy. Soc. London (A) 193 (1948), 260-281.

Applied Mathematics Branch, Mechanics Division, U. S. Naval Research LABORATORY. 


\title{
PACIFIC JOURNAL OF MATHEMATICS
}

\section{EDITORS}

H. L. RoYden

Stanford University

Stanford, California

\section{R. A. Beaumont}

University of Washington

Seattle 5 , Washington
A. L. Whiteman

University of Southern California

Los Angeles 7, California

E. G. Straus

University of California

Los Angeles 24, California

\section{ASSOCIATE EDITORS}

\author{
E. F. BECKENBACH \\ C. E. BURGESS \\ M. HALL \\ E. HEWITT
}
A. HORN
V. GANAPATHY IYER
R. D. JAMES
M. S. KNEBELMAN

L. NACHBIN

I. NIVEN

T. G. OSTROM

M. M. SCHIFFER
G. SZEKERES

F. WOLF

K. YOSIDA

\section{SUPPORTING INSTITUTIONS}

\author{
UNIVERSITY OF BRITISH COLUMBIA \\ CALIFORNIA INSTITUTE OF TECHNOLOGY \\ UNIVERSITY OF CALIFORNIA \\ MONTANA STATE UNIVERSITY \\ UNIVERSITY OF NEVADA \\ OREGON STATE COLLEGE \\ UNIVERSITY OF OREGON \\ UNIVERSITY OF SOUTHERN CALIFORNIA
}

Mathematical papers intended for publication in the Pacific Journal of Mathematics should be typewritten (double spaced), and the author should keep a complete copy. Manuscripts may be sent to any of the editors. All other communications to the editors should be addressed to the managing editor, E. G. Straus at the University of California, Los Angeles 24, California.

50 reprints per author of each article are furnished free of charge; additional copies may be obtained at cost in multiples of 50 .

The Pacific Journal of Mathematics is published quarterly, in March, June, September, and December. The price per volume (4 numbers) is $\$ 12.00$; single issues, $\$ 3.50$. Back numbers are available. Special price to individual faculty members of supporting institutions and to individual members of the American Mathematical Society: $\$ 4.00$ per volume; single issues, $\$ 1.25$.

Subscriptions, orders for back numbers, and changes of address should be sent to Pacific Journal of Mathematics, 2120 Oxford Street, Berkeley 4, California.

Printed at Kokusai Bunken Insatsusha (International Academic Printing Co., Ltd.), No. 10, 1-chome, Fujimi-cho, Chiyoda-ku, Tokyo, Japan.

PUBLISHED BY PACIFIC JOURNAL OF MATHEMATICS, A NON-PROFIT CORPORATION

The Supporting Institutions listed above contribute to the cost of publication of this Journal, but they are not owners or publishers and have no responsibility for its content or policies. 


\section{Pacific Journal of Mathematics}

\section{Vol. 7, No. 4 \\ April, 1957}

Robert Geroge Buschman, A substitution theorem for the Laplace

transformation and its generalization to transformations with

symmetric kernel .

S. D. Conte, Numerical solution of vibration problems in two space variables ...................................... 1535

Paul Dedecker, A property of differential forms in the calculus of variations ......................................... 1545

H. Delange and Heini Halberstam, A note on additive functions . . . . . . . 1551

Jerald L. Ericksen, Characteristic direction for equations of motion of non-Newtonian fluids ................................. 1557

Avner Friedman, On two theorems of Phragmén-Lindelöf for linear elliptic and parabolic differential equations of the second order ........... 1563

Ronald Kay Getoor, Additive functionals of a Markov process . . . . . . . . . 1577

U. C. Guha, $(\gamma, k)$-summability of series . . . . . . . . . . . . . . . . . . 1593

Alvin Hausner, The tauberian theorem for group algebras of vector-valued functions ...................................... 1603

Lester J. Heider, $T$-sets and abstract $(\mathrm{L})$-spaces ................. 1611

Melvin Henriksen, Some remarks on a paper of Aronszajn and

Panitchpakdi........................................ 1619

H. M. Lieberstein, On the generalized radiation problem of A. Weinstein ... 1623

Robert Osserman, On the inequality $\Delta u \geq f(u) \ldots \ldots \ldots \ldots \ldots \ldots \ldots . \ldots 1641$

Calvin R. Putnam, On semi-normal operators . . . . . . . . . . . . . . . . . . 1649

Binyamin Schwarz, Bounds for the principal frequency of the

non-homogeneous membrane and for the generalized Dirichlet integral...

Edward Silverman, Morrey's representation theorem for surfaces in metric

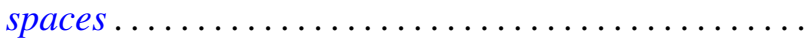

V. N. Singh, Certain generalized hypergeometric identities of the Rogers-Ramanujan type. II .

R. J. Smith, A determinant in continuous rings 1701

Drury William Wall, Sub-quasigroups of finite quasigroup 1711

Sadayuki Yamamuro, Monotone completeness of normed semi-ordered linear spaces.

C. T. Rajagopal, Simplified proofs of "Some Tauberian theorems" of Jakimovski: Addendum and corrigendum ............

N. Aronszajn and Prom Panitchpakdi, Correction to: "Extension of uniformly continuous transformations in hyperconvex metric spaces"...................................

Alfred Huber, Correction to: "The reflection principle for polyharmonic functions" 